\title{
28 Research Square \\ Designing and Modeling of a Municipal Wastewater Treatment Plant With GPS-X
}

Fahim Shahriar Sakib ( $\nabla$ fssakib98@gmail.com )

Utility Professionals https://orcid.org/0000-0001-6002-714X

\section{Research Article}

Keywords: Municipal Wastewater, Sewage Treatment Plant, Safe disposal, Modeling, Removal efficiency

Posted Date: January 6th, 2022

DOI: https://doi.org/10.21203/rs.3.rs-1209601/v1

License: (9) This work is licensed under a Creative Commons Attribution 4.0 International License. Read Full License 


\section{Abstract}

Wastewater treatment is the process used to remove contaminants from wastewater to produce an effluent suitable for discharge to the environment. Several kinds of wastewater are treated based on the concentration of various parameters. Domestic Wastewater/Municipal Wastewater is treated in Sewage Treatment Plants (STP), which involve various types of processes like physical, chemical and biological to treat the wastewater better. The treatment plant involves five steps of the process - preliminary treatment, primary treatment, secondary treatment, tertiary treatment and sludge treatment to meet the safe disposal guidelines of the department of the environment. In this paper, a municipal wastewater treatment plant for Uttara City is designed and modeled with GPS-X. GPS-X is a wastewater treatment plant modeling software package that is used for verify the treatment process. The design calculation of all unit operations is shown with a specification table. The Process Flow Diagram (PFD), Process Block Diagram (PBD) and mass balance of the process are expressed in the article. The design calculation of the STP is performed with a steady-state process. After each unit operation, the concentration of various parameters - TSS, BOD, VSS, TN is analyzed and the removal efficiency for different equipment is calculated. The STP is designed to maintain the guidelines of the Department of Environment, Bangladesh. At the end of the paper, the designing and modeling steps of a sewage treatment plant will be pursued. Finally, some recommendations are done in the conclusion section.

\section{Introduction}

Wastewater/Sewage is a polluted form of water that includes human wastes, organic substances, oil and greases, chemicals, etc. They are categorized by the manner - domestic wastewater, industrial wastewater, storm wastewater (Metcalf et al., 2003; Patterson, 1985; Abdel-Raouf, 2012). Wastewater treatment is a process that is used to remove pollutants to get the clear effluent from the waste influent (Sonune \& Ghate, 2004; Libralato, 2012; Speece, 1983). A wastewater/sewage treatment plant is designed according to the nature and composition of the contaminants in the wastewater (Droste \& Gehr, 2018; Erckenfelder Jr, 2000; Pescod, 1992). The developed countries are establishing new technologies and processes on wastewater treatment or water treatment. However, the developing and under developed countries are facing difficulties for initiating the required infrastructure for wastewater treatment. Problem of improper wastewater treatment facilities is severe in highly dense countries like Bangladesh. There are not enough planning and program from Government of Bangladesh (GoB) in wastewater treatment. At present, deficiency in budget, improper design and treatment of most of existing plants, unavailability of professionals, engineers, and skilled operators, and insufficient public awareness about the risk of direct discharge of wastewater to water channels had led to serious deficiencies in operations in the country's wastewater treatment plants. Due to unavailability of skilled operator and budget, no preventive measure is taken in the wastewater treatment plant. Improper process and instrumentation design sometimes led to a severe environmental problem in the country.

Domestic wastewater/Municipal wastewater is treated in a Sewage Treatment Plant (STP) which involves different types of processes like chemical, physical and biological to treat the sewage effectively 
(Crini \& Lichtfouse, 2019; Massoud et al., 2009). In the context of Bangladesh, most of the treatment plant is operated with the physical and biological process. The treatment process includes five steps: preliminary treatment, primary treatment, secondary treatment, tertiary treatment and sludge treatment (Russell, 2019; Marco et al., 1997). Preliminary treatment is the physical process, primary treatment is the physical process or the combination of physical and chemical processes, secondary treatment is the combination of physical and biological processes, tertiary treatment is the combination of physical and chemical process and sludge treatment is the combination of a physical and chemical process (Cheremisinoff, 2001; Mara, 2013; Schroeder, 1977). The selection of processes depends on the safe disposable guideline of the Department of Environment - DoE (DoE, 1997).

The objective of this study to design a large wastewater treatment plant for Uttara city near the capital of Bangladesh and verify the treatment processes. Designing a large scale STP is quite challenging for the students, academics, engineers and process designers. This study will help to design a large scale STP in newly developing and under developed countries. Improper and inexperienced design can be fatal for environment. Also, the paper describes treatment processes and all unit operations. The plant process is a combination of physical and biological processes. The treatment process is designed following the discharge guideline of ECR 97. The detailed specification of unit operations is developed in the paper. The STP is modeled with GPS- $X$, a process simulation software which is developed by Hydromantis. The process block diagram (PBD), process flow diagram (PFD) is developed with mass balance in a Sanky diagram. The modeling and designing are performed with a steady-state process. After each unit operation, the concentration of various parameters - Total Suspended Solids (TSS), Biological Oxygen Demand (BOD), Volatile Suspended Solids (VSS), Total Nitrogen (TN) is analyzed and shown. Finally, the removal efficiencies of different unit operations are calculated to show their performance in the treatment process.

\section{Methodology \\ 2.1 Location}

The wastewater treatment plant was designed to treat the sewage of the whole Uttara thana in the Dhaka metropolitan area, Bangladesh. The city is located in between $23^{0} 51^{\prime}$ and $23^{0} 52^{\prime}$ north latitudes and in between $90^{\circ} 22^{\prime}$ and $90^{\circ} 24^{\prime}$ east longitudes. Uttara city is one of the developing cities in Dhaka. The city is surrounded by Gazipur Sadar Upazila on the north, Airport and Pallabi thanas on the south, Dakshinkhan thana on the east, Pallabi and Turag thanas on the west. The climate of the location is very much important to design a treatment plant. December - February is the winter period in Dhaka and March November is the summer period. May - September is the wet period and October - April is the dry period. The temperature and rainfall affect the capacity load of the STP. Fig. 1 shows the map of the Uttara city rounded with a red color mark.

\subsection{Capacity Calculation}


The STP design process mainly depends on the load of the wastewater and the constituents of the pollutants (Vesilind, 2003). The sewage treatment plant was designed to cover the whole area of Uttara Thana situated in the capital of Bangladesh. The total population of the Uttara Thana was 66,636 in 2001 (BBS, 2003). We can get the present population number using a statistical equation, Eq. 1. (Samal, 2016).

$$
P=P_{0} e^{(0.05 \times n)}
$$

1

$$
Q=P \times C
$$

2

$$
Q_{P}=Q \times P_{f}
$$

3

Here, $\mathrm{P}=$ Population, $\mathrm{P}_{0}=$ New population, $\mathrm{n}=$ Number of years, $\mathrm{Q}=$ Water demand, $\mathrm{C}=$ Water consumption per capita per day, $Q_{P}=$ Peak demand and $P_{f}=$ Peak factor.

The STP was designed considering for the 2041 year, 20 years ahead of 2021. The initial design stage is considered for 2021. That means the STP is designed for 20 years. The water consumption was taken as 200 liters per capita per day with full capacity as a reference from Bangladesh National Code (BNBC 2020). Water demand is the product of population and water consumption per capita. The peak factor was taken 3 for preventing malfunctioning in high load operation situations (Samal, 2016). Every STP has to be designed under the consideration of peak time flow. Table 1 represents the population, future population after 10 years, current water demand and the peak flow rate of the STP. Therefore, the average capacity of the STP is $98475.4 \mathrm{~m}^{3} / \mathrm{d}$ or 98.5 million liters per day (MLD) and the peak time capacity is $295426 \mathrm{~m}^{3} / \mathrm{d}$ or 295.5 million liters per day (MLD).

Table 1

Sewage Treatment Plant Capacity Calculation

\begin{tabular}{|llllll|}
\hline Parameters & 2001 year & 2011 year & 2021 year & 2031 year & 2041 year \\
\hline Population & 66636 & 109864 & 181135 & 298642 & 492377 \\
\hline Population after 10 years & 109864 & 181135 & 298642 & 492377 & 811793 \\
\hline Water demand $\left(\mathrm{m}^{3} / \mathrm{d}\right), \mathrm{Q}$ & 13327.2 & 21972.8 & 36227.1 & 59728.4 & 98475.4 \\
\hline Peak flow $\left(\mathrm{m}^{3} / \mathrm{d}\right), \mathrm{Q}_{\mathrm{P}}$ & 39981.6 & 65918.5 & 108681 & 179185 & 295426 \\
\hline
\end{tabular}

\subsection{Influent and Effluent Characteristics}


The wastewater/sewage is black-colored water with high turbidity and human excreta (Kumar et al 2010). The characteristics data of influent sewage and effluent quality are shown in Table 2 . The effluent sewage discharge limits are taken from ECR, 97. The design was performed considering the strong concentration of influent sewage (Metcalf et al., 2003). After the treatment process, the effluent becomes clean and clear. Effluent will be discharged to the inland surface water. The process design and selection are primarily depended on the constituents of the wastewater.

Table 2

Different Parameters Concentrations of Influent and Effluent

\begin{tabular}{|lll|}
\hline Parameters & Influent $(\mathrm{mg} / \mathrm{l})$ & Effluent $(\mathrm{mg} / \mathrm{l})$ \\
\hline TSS & $<400$ & $<150$ \\
\hline TDS & $<860$ & $<2100$ \\
\hline BOD & $<350$ & $<50$ \\
\hline TN & $<70$ & $<100$ \\
\hline (Source: Metcalf et al., 2003; ECR, 1997) \\
\hline
\end{tabular}

\subsection{Process Flow Description}

Sewage from different apartments is coming through the gravity sewer to the pumping station. The vertical lift pumps are placed to pump the black wastewater to the treatment plant. The sewage is transferred to the equalization tank. An equalization tank is used to fix the diurnal variable flow rate in the treatment plant. Variable flow rate can affect the effluent quality and process performance of the plant (LaGrega and Keenan, 1974). From the equalization tank, the sewage is transferred through the bar screen chamber and grit chamber. Bar screen is used to remove rags, plastics, cans, etc. and grit chamber removes sands, gravels, small rocks (Vesilind, 2003; Sawicki, 2004). These are called preliminary treatments which are physical processes. After the preliminary process, the primary treatment process occurs through the primary sedimentation tank. Primary sedimentation tank removes suspended particles and organic matters by gravity with chemicals or without chemicals (Kumar et al., 2010). Microorganisms help to remove BOD by oxidizing organic matters with the presence of oxygen in the air and settled in the secondary clarifier by growing into larger flocs (Gebara, 1999). Part of the sludge is recycled to the aeration tank to improve the performance of the process which is called the activated sludge process (Mittal, 2011). The disinfection process happens after the secondary treatment. Chlorination, UV or ozonation is used to remove harmful pathogens (Lazarova et al., 1999). In this design, a chlorination tank is introduced for disinfection. Sludge treatment is essential for a sewage treatment plant. Thickener is used to thicken the primary sludge (PS), waste activated sludge (WAS) or mixed sludge. Gravity thickener, dissolved air flotation, centrifugal, rotary drum thickening is used according to the types and characteristics (Patterson, 1985). Following the thickening process, digesting process is introduced. Digester helps to digest sludge anaerobically or aerobically to reduce sludge load, kill pathogens and stabilize the sludge (Andreoli et al., 2007). Finally, sludge dewatering is introduced to dewater the sludge 
and form cake to reduce the sludge handling cost (Mahmoud, 2013). The pumping station is not shown in the paper as it does not have any impact in the process. In this plant, gravity thickener is designed to thicken the mixed sludge, the combination of PS and WAS. Total sludge is mixed with the dilution water from the discharge line to maintain the hydraulic loading rate. A single anaerobic digester is introduced to digest the sludge anaerobically to get the by-product methane gas and the digested sludge is dewatered in the belt press filter. The press filter will convert the sludge into the cake and the filtrate will be transferred to the equalization tank again. The treated wastewater will be discharged to the Turag River and the caked sludge will be taken by municipal sludge truck. Fig. 2 shows the overall process block diagram (PBD) of the sewage treatment plant.

\section{Design Calculation}

\subsection{Equalization Tank}

Assuming number of tanks, $\mathrm{N}=4$ Nos.

Flow rate for each tank, $\mathrm{Q}=\frac{Q_{P}}{N}=\frac{295.5}{4}=73.9 \mathrm{MLD}$

Assuming detention time, $\mathrm{T}=2$ hours

Volume, $\mathrm{V}=Q \times T=73.9 \times 0.5=6145.7 \mathrm{~m}^{3}$

Assuming depth, $\mathrm{H}=5 \mathrm{~m}$ (excluding $0.5 \mathrm{~m}$ free board) and width, $\mathrm{W}=30 \mathrm{~m}$

Length, $\mathrm{L}=\frac{V}{H \times W}=\frac{6154.7}{5 \times 30}=41 \mathrm{~m}$

Air requirement for mixing, $Q_{A}=V \times a=6145.7 \times 1.1=6760.3 \mathrm{~m}^{3} / \mathrm{h} \sim 6761 \mathrm{~m}^{3} / \mathrm{h}$

[Mixing factor, $\left.a=1.1 \mathrm{~m}^{3} / \mathrm{m}^{3}\right]$ (Kodavasal, 2011)

Assuming minimum air flux of diffuser, $F_{A}=12 \mathrm{~m}^{3} /$ hour (Kodavasal, 2011)

Number of diffusers, $\mathrm{N}_{\mathrm{D}}=\frac{Q_{A}}{N_{D}}=\frac{6761}{12}=563.4 \sim 564$ Nos.

Specification of equalization tanks are shown in Table 3. 
Table 3

Specification of equalization tank

\begin{tabular}{|ll|}
\hline Parameter & Value \\
\hline Quantity & 4 Nos. \\
\hline Flow Rate & 73.9 MLD \\
\hline Detention Time & 2 hours \\
\hline Volume & $6145.7 \mathrm{~m}^{3}$ \\
\hline Length & $41 \mathrm{~m}$ \\
\hline Width & $30 \mathrm{~m}$ \\
\hline Height & $5 \mathrm{~m}$ \\
\hline Free Board & $0.5 \mathrm{~m}$ \\
\hline Air Requirement & $6761 \mathrm{~m}^{3} / \mathrm{h}$ \\
\hline Diffuser Quantity & $564 \mathrm{Nos}^{\circ}$ \\
\hline
\end{tabular}

\subsection{Bar Screen Chamber}

Two screens will be used. Coarse screen precedes medium screen.

Coarse Screen: Assuming,

Length, $L=1000 \mathrm{~mm}$, Width, $\mathrm{W}=10 \mathrm{~mm}$,

Opening, $0=50 \mathrm{~mm}$, Inclination, I = 45 Degree. (Metcalf et al., 2003)

Assuming number of bar screen chambers, $\mathrm{N}=4$ Nos.

Flow rate for each tank, $\mathrm{Q}=\frac{Q_{P}}{N}=\frac{295.5}{4}=73.9 \mathrm{MLD}=\frac{73900}{24 \times 3600}=0.86 \mathrm{~m}^{3} / \mathrm{s}$

Velocity into screen, $v_{i}=0.6 \mathrm{~m} / \mathrm{s}$

Cross-section area of channel, $\mathrm{A}_{\mathrm{C}}=\frac{Q}{V_{i}}=\frac{0.86}{0.6}=1.43 \mathrm{~m}^{2}$

Gross area of channel, $\mathrm{A}_{\mathrm{G}}=A_{C} \times\left(1+\frac{W}{O}\right)=1.43 \times\left(1+\frac{10}{50}\right)=1.72 \mathrm{~m}^{2}$

Velocity above screen, $\mathrm{v}_{\mathrm{o}}=v_{i} \times \frac{O}{O+W}=0.6 \times \frac{50}{(10+50)}=0.5 \mathrm{~m} / \mathrm{s}$

Bar Shape Factor, B = 2.42 [MS Flat Sheet Bar] 
Head loss, $\mathrm{h}_{\mathrm{L}}=B \times{\frac{W^{4}}{o}}^{/ 3} \times \frac{v_{i}^{2}}{2 g}$

(4) (Varadi, 1984)

$=2.42 \times\left(\frac{10}{50}\right)^{4 / 3} \times \frac{0.6^{2}}{2 \times 9.81} \times \sin 45=0.35 \mathrm{~m}$

Assuming submergence depth, $\mathrm{H}=1 \mathrm{~m}$

Width of the channel, $\mathrm{W}_{\mathrm{C}}=\frac{{ }^{A_{G}}}{H}=\frac{1.72}{1}=1.72 \mathrm{~m}=1720 \mathrm{~mm}$

Number of bars, $\mathrm{N}_{B}=\frac{1720}{(50+10)}=28.5 \sim 29$ Nos.

Medium Screen: Assuming,

Length, $\mathrm{L}=1000 \mathrm{~mm}$, Width, $\mathrm{W}=10 \mathrm{~mm}$,

Opening, $0=20$ mm, Inclination, I = 60 Degree. (Metcalf et al., 2003)

According to the previous calculations,

Cross-section area of channel, $A_{C}=1.42 \mathrm{~m}_{2}$

Gross area of channel, $A_{G}=2.14 \mathrm{~m}_{2}$

Velocity above screen, $\mathrm{v}_{\mathrm{o}}=0.4 \mathrm{~m} / \mathrm{s}$

Head loss, $h_{L}=1.5 \mathrm{~m}$

Assuming submergence depth, $\mathrm{H}=1 \mathrm{~m}$

Width of the channel, $\mathrm{W}_{\mathrm{C}}=2.14 \mathrm{~m}=2140 \mathrm{~mm}$

Number of bars, $\mathrm{N}_{\mathrm{B}}=71.2 \sim 72$ Nos.

Specification of bar screen chambers are shown in Table 4. 
Table 4

Specification of bar screen chamber

\begin{tabular}{|lll|}
\hline Parameter & Coarse Screen & Medium Screen \\
\hline Quantity of Screen Chamber & 4 Nos. & 4 Nos. \\
\hline Flow Rate & $73.9 \mathrm{MLD}$ & $73.9 \mathrm{MLD}$ \\
\hline Length of Bar & $1000 \mathrm{~mm}$ & $1000 \mathrm{~mm}$ \\
\hline Width of Bar & $10 \mathrm{~mm}$ & $10 \mathrm{~mm}$ \\
\hline Opening & $50 \mathrm{~mm}$ & $20 \mathrm{~mm}$ \\
\hline Inclination & $45 \mathrm{degrees}$ & $60 \mathrm{degrees}$ \\
\hline Cross-section Area of Channel & $1.43 \mathrm{~m}^{2}$ & $1.42 \mathrm{~m}^{2}$ \\
\hline Gross Area of Channel & $1.72 \mathrm{~m}^{2}$ & $2.14 \mathrm{~m}^{2}$ \\
\hline Head Loss & $0.35 \mathrm{~m}$ & $1.5 \mathrm{~m}$ \\
\hline Submergence Depth & $1 \mathrm{~m}$ & $1 \mathrm{~m}$ \\
\hline Width of Channel & $1.72 \mathrm{~m}$ & $2.14 \mathrm{~m}$ \\
\hline Quantity of Bar & $29 \mathrm{Nos}$. & $72 \mathrm{Nos}$. \\
\hline
\end{tabular}

\subsection{Grit Chamber}

Assuming number of horizontal grit chambers, $\mathrm{N}=8$ Nos. for periodic cleaning.

Flow rate for each tank, $\mathrm{Q}=\frac{Q_{P}}{N}=\frac{295.5}{8}=36.95 \mathrm{MLD}$

Plant operating time, $T_{0}=24$ hours in a day.

Assuming detention time, $\mathrm{T}=180$ seconds.

Assuming surface loading rate, $L=750 \mathrm{~m}^{3} / \mathrm{m}^{2}$.day

Surface area, $\mathrm{A}=\frac{Q}{L}=\frac{36950}{750}=49.24 \mathrm{~m}^{2}$

Diameter, $\mathrm{D}=\sqrt{\frac{4 A}{\Pi}}=\sqrt{\frac{4 \times 49.24}{\Pi}}=8 \mathrm{~m}$

Volume, $\mathrm{V}=\frac{Q \times T}{T_{O} \times 3600}=\frac{36950 \times 180}{24 \times 3600}=77 \mathrm{~m}^{3}$ 
Side wall height, $\mathrm{H}=\frac{V}{A}=\frac{77}{49.24}=1.56 \sim 1.6 \mathrm{~m}$

Bottom slope factor, $S_{F}=0.083$ (Metcalf et al., 2003)

Bottom slope, $\mathrm{S}_{\mathrm{L}}=H \times S_{F}=1.6 \times 0.083=0.13 \mathrm{~m}$

Center height, $\mathrm{H}_{\mathrm{C}}=H+S_{L}=1.6+0.13=1.73 \sim 1.8 \mathrm{~m}$

Specification of grit chambers are shown in Table 5.

Table 5

Specification of grit chamber

\begin{tabular}{|ll|}
\hline Parameter & Value \\
\hline Quantity & 8 Nos. \\
\hline Flow Rate & $36.95 \mathrm{MLD}$ \\
\hline Detention Time & 3 minutes \\
\hline Surface Loading Rate & $750 \mathrm{~m}^{3} / \mathrm{m}^{2}$.day \\
\hline Diameter & $8 \mathrm{~m}$ \\
\hline Height & $1.6 \mathrm{~m}$ \\
\hline Center Height & $1.8 \mathrm{~m}$ \\
\hline Free Board & $0.5 \mathrm{~m}$ \\
\hline
\end{tabular}

\subsection{Primary Clarifier}

Assuming overflow rate, $\mathrm{O}_{\mathrm{A}}=40 \mathrm{~m}^{3} / \mathrm{m}^{2}$.day for average flow. So, average flowrate of the sewage will be used for calculation.

Assuming number of primary clarifiers, $\mathrm{N}=4$ Nos.

Average flow rate for each clarifier, $\mathrm{Q}_{\mathrm{A}}=\frac{Q_{P}}{N}=\frac{98.5}{4}=24.62 \mathrm{MLD}$

Detention time, $\mathrm{T}=2.5$ hours.

Surface area, $\mathrm{A}=\frac{Q_{A}}{O_{A}}=\frac{24620}{40}=615.5 \mathrm{~m}^{2}$ 
Diameter, $\mathrm{D}=\sqrt{\frac{\overline{4 A}}{\Pi}}=\sqrt{\frac{4 \times 615.5}{\Pi}}=28 \mathrm{~m}$

Volume, $\mathrm{V}=\frac{Q_{A} \times T}{T_{O}}=\frac{24620 \times 2.5}{24}=2564.5 \mathrm{~m}^{3}$

Side wall height, $\mathrm{H}=\frac{V}{A}=\frac{2564.5}{615.5}=4.17 \sim 4.2 \mathrm{~m}$

Bottom slope factor, $S_{F}=0.083$ (Metcalf et al., 2003)

Bottom slope, $\mathrm{S}_{\mathrm{L}}=H \times S_{F}=4.2 \times 0.083=0.35 \mathrm{~m}$

Center height, $\mathrm{H}_{\mathrm{C}}=H+S_{L}=4.2+0.35=4.55 \mathrm{~m}$

Weir loading, $W_{L}=250 \mathrm{~m}^{3} / \mathrm{m}^{2}$.day (Metcalf et al., 2003)

Scour Velocity, $\mathrm{V}_{\mathrm{H}}=\sqrt{\left(\frac{8 k(s-1) g d}{f}\right)}$

$=\left(\frac{8 \times 0.05 \times 0.25 \times 9.81 \times\left(100 \times 10^{-6}\right)}{0.025}\right)^{1 / 2}=0.063 \mathrm{~ms}^{-1}$

Cohesion Constant, $\mathrm{k}=0.05$

Specific Gravity, $\mathrm{s}=1.25$

Acceleration due to gravity, $\mathrm{g}=9.8 \mathrm{~ms}^{-2}$

Diameter of Particles, $d=100 \mu \mathrm{m}$

Darcy-Weisbach Friction Factor, $f=0.025$

Specification of primary clarifiers are shown in Table 6. 
Table 6

Specification of primary clarifier

\begin{tabular}{|ll|}
\hline Parameter & Value \\
\hline Quantity & 4 Nos. \\
\hline Flow Rate & $24.62 \mathrm{MLD}$ \\
\hline Detention Time & 2.5 hours \\
\hline Overflow Rate at Average Flowrate & $40 \mathrm{~m}^{3} / \mathrm{m}^{2}$.day \\
\hline Overflow Rate at Peak Flowrate & $120 \mathrm{~m}^{3} / \mathrm{m}^{2}$.day \\
\hline Surface Area & $615.5 \mathrm{~m}^{2}$ \\
\hline Diameter & $28 \mathrm{~m}$ \\
\hline Side Wall Height & $4.2 \mathrm{~m}$ \\
\hline Center Height & $4.55 \mathrm{~m}$ \\
\hline Free Board & $0.5 \mathrm{~m}$ \\
\hline Weir Loading & $250 \mathrm{~m}^{3} / \mathrm{m}^{2}$.day \\
\hline
\end{tabular}

\subsection{Aeration Tank}

Assuming number of aeration tanks, $\mathrm{N}=8$ Nos.

Flow rate for each tank, $\mathrm{Q}=\frac{Q_{P}}{N}=\frac{295.5}{8}=36.95 \mathrm{MLD}$

Inlet BOD, $Y_{1}=350 \mathrm{mg} / \mathrm{l}$ and outlet BOD, $Y_{0}=35 \mathrm{mg} / \mathrm{l}$

[The underflow rate and the BOD removal of the primary clarifier are neglected to get the better performance]

Efficiency, $\eta=\frac{Y_{I}-Y_{O}}{Y_{I}}=\frac{350-35}{350}=90 \%$

Assuming, $\mathrm{F} / \mathrm{M}=0.12$ and $\mathrm{MLSS}=3500 \mathrm{mg} / \mathrm{l}($ Samal, 2016)

Volume, $\mathrm{V}=\frac{Q \times Y_{I}}{F / M \times M L S S}=\frac{36950 \times 350}{0.12 \times 3500}=30791.67 \mathrm{~m}^{3}$

Assuming Width, $\mathrm{W}=70 \mathrm{~m}$ and Depth, $\mathrm{H}=5.5 \mathrm{~m}$ [Excluding $0.5 \mathrm{~m}$ free-board]

Length, $\mathrm{L}=\frac{V}{W \times H}=\frac{30791.67}{70 \times 5.5}=80 \mathrm{~m}$ 
Aeration Period, $\mathrm{T}_{\mathrm{A}}=\frac{30791.67 \times 24}{36950}=20$ hours.

Solids Retention Time, SRT

$$
V \times M L S S=\frac{\alpha \times Q\left(Y_{I}-Y_{O}\right) \times S R T}{1+K \times S R T}
$$

6

$[a=1.0$ and $K=0.06]$

$$
30791.67 \times 3500=\frac{1 \times 36950 \times(350-35) \times S R T}{1+0.06 \times S R T}
$$

$\mathrm{SRT}=20.83 \sim 21$ days

BOD load $/$ day, $Y_{D}=(Q \times 1000) \times \frac{Y_{I}}{10^{6}}=(36950 \times 1000) \times \frac{350}{10^{6}}=12932.5 \mathrm{~kg} / \mathrm{day}$

BOD load $/$ hour, $\mathrm{Y}_{\mathrm{H}}=\frac{Y_{D}}{T_{A}}=\frac{12932.5}{20}=646.63 \mathrm{~kg} / \mathrm{hour}$

Air requirement for $\mathrm{BOD}, \mathrm{A}_{\mathrm{B}}=Y_{H} \times 115=646.63 \times 115=74362.5 \sim 74363 \mathrm{~m}^{3} / \mathrm{h}($ Kodavasal, 2011$)$

Air requirement for mixing, $A_{M}=V \times 1.1=30791.67 \times 1.1=33870.84 \mathrm{~m}^{3} \sim 33871 \mathrm{~m}^{3} /$ day

As air requirement for BOD is greater than for mixing, higher value will be used for further calculation

Minimum air flux of diffuser, $A_{F}=12 \mathrm{~m}^{3} /$ hour

Number of diffusers, $\mathrm{N}_{\mathrm{D}}=\frac{A_{M}}{A_{F}}=\frac{74363}{12}=6196.9 \sim 6197$ Nos.

Specification of aeration tanks are shown in Table 7. 
Table 7

Specification of aeration tank

\begin{tabular}{|ll|}
\hline Parameter & Value \\
\hline Quantity & 8 Nos. \\
\hline Flow Rate & $36.95 \mathrm{MLD}$ \\
\hline Aeration Period & 20 hours \\
\hline Length & $80 \mathrm{~m}$ \\
\hline Width & $70 \mathrm{~m}$ \\
\hline Depth & $5.5 \mathrm{~m}$ \\
\hline Free Board & $0.5 \mathrm{~m}$ \\
\hline Solids Retention Time & 21 days \\
\hline Air Requirement & $81799 \mathrm{~m}_{3} /$ day \\
\hline Air Flux of Diffuser & $12 \mathrm{~m}^{3} /$ hour \\
\hline Number of diffusers & 6197 Nos. \\
\hline
\end{tabular}

\subsection{Secondary Clarifier}

Assuming overflow rate, $\mathrm{O}_{\mathrm{A}}=24 \mathrm{~m}^{3} / \mathrm{m}^{2}$.day for average flow. So, average flowrate of the sewage will be used for calculation.

Assuming number of secondary clarifiers, $\mathrm{N}=4$ Nos.

Average flow rate for each clarifier, $\mathrm{Q}_{\mathrm{A}}=\frac{Q_{P}}{N}=\frac{98.5}{4}=24.62 \mathrm{MLD}$

Assuming residual MLSS in clarifier, $\mathrm{MLSS}_{\mathrm{C}}=16000$

Recycle ratio, $\mathrm{R}=\frac{M L S S}{M L S S_{C}-M L S S}=\frac{3500}{16000-3500}=0.28$

Total inflow, $\mathrm{Q}_{\mathrm{T}}=Q \times(1+R)=24.62 \times(1+0.28)=31.51 \mathrm{MLD}$

Detention time, $\mathrm{T}=2$ hours.

Surface area, $\mathrm{A}=\frac{Q_{A}}{O_{A}}=\frac{31510}{24}=1312.9 \mathrm{~m}^{2}$ 
Diameter, $\mathrm{D}=\sqrt{\frac{\overline{4 A}}{\Pi}}=\sqrt{\frac{4 \times 1312.9}{\Pi}}=40.9 \sim 41 \mathrm{~m}$

Volume, $\mathrm{V}=\frac{Q_{A} \times T}{T_{O}}=\frac{31510 \times 2}{24}=2625.83 \mathrm{~m}^{3}$

Side wall height, $\mathrm{H}=\frac{V}{A}=\frac{2625.83}{1312.9}=2 \mathrm{~m}$

Bottom slope factor, $S_{F}=0.083$ (Metcalf et al., 2003)

Bottom slope, $\mathrm{S}_{\mathrm{L}}=H \times S_{F}=2 \times 0.083=0.17 \sim 0.2 \mathrm{~m}$

Center height, $\mathrm{H}_{\mathrm{C}}=H+S_{L}=2+0.2=2.2 \mathrm{~m}$

Weir loading, $W_{L}=250 \mathrm{~m}^{3} / \mathrm{m}^{2}$.day (Metcalf et al., 2003)

Specification of secondary clarifiers are shown in Table 8.

Table 8

Specification of secondary clarifier

\begin{tabular}{|ll|}
\hline Parameter & Value \\
\hline Quantity & 4 Nos. \\
\hline Flow Rate & $31.51 \mathrm{MLD}$ \\
\hline Detention Time & 2 hours \\
\hline Overflow Rate at Average Flowrate & $24 \mathrm{~m}^{3} / \mathrm{m}^{2}$.day \\
\hline Overflow Rate at Peak Flowrate & $72 \mathrm{~m}^{3} / \mathrm{m}^{2}$.day \\
\hline Surface Area & $1312.9 \mathrm{~m}^{2}$ \\
\hline Diameter & $41 \mathrm{~m}$ \\
\hline Side Wall Height & $2 \mathrm{~m}$ \\
\hline Center Height & $2.2 \mathrm{~m}$ \\
\hline Free Board & $0.5 \mathrm{~m}$ \\
\hline Weir Loading & $250 \mathrm{~m}^{3} / \mathrm{m}^{2}$.day \\
\hline
\end{tabular}

\subsection{Chlorination Chamber}

Assuming number of chlorination chambers, $\mathrm{N}=4$ Nos. 
Flow rate for each tank, $\mathrm{Q}=\frac{Q_{P}}{N}=\frac{295.5}{4}=73.9 \mathrm{MLD}$

Chlorine contacts time, $T=0.5$ hours (Maximum contact time) (Metcalf et al., 2003)

Volume, $\mathrm{V}=\frac{Q \times T}{T_{O}}=\frac{73900 \times 0.5}{24}=1539.6 \mathrm{~m}^{3}$

Assuming height, $\mathrm{H}=3 \mathrm{~m}$ and width, $\mathrm{W}=20 \mathrm{~m}$.

Length, $\mathrm{L}=\frac{V}{H \times W}=\frac{1539.6}{3 \times 20}=25.6 \sim 26 \mathrm{~m}$

Assuming maximum required chlorine dosage, $\mathrm{C}_{\mathrm{Cl}, \mathrm{p}}=20 \mathrm{mg} / \mathrm{L}$

Chlorinator capacity, $\mathrm{M}_{\mathrm{P}}=\frac{Q \times C_{C l, p}}{1000}=\frac{73900 \times 20}{1000}=1478 \mathrm{~kg} / \mathrm{day}$

Assuming average chlorine dosage, $\mathrm{C}_{\mathrm{Cl}, \mathrm{a}}=10 \mathrm{mg} / \mathrm{L}$

Daily consumption of chlorine, $\mathrm{M}_{\mathrm{P}}=\frac{Q_{A} \times C_{C l, a}}{1000}=\frac{24620 \times 10}{1000}=246.2 \mathrm{~kg} /$ day

Specification of chlorination chambers are shown in Table 9.

Table 9

Specification of chlorination chamber

\begin{tabular}{|ll|}
\hline Parameter & Value \\
\hline Quantity & 4 Nos. \\
\hline Flow Rate & $73.9 \mathrm{MLD}$ \\
\hline Aeration Period & 0.5 hour \\
\hline Length & $26 \mathrm{~m}$ \\
\hline Width & $20 \mathrm{~m}$ \\
\hline Height & $3 \mathrm{~m}$ \\
\hline Free Board & $0.5 \mathrm{~m}$ \\
\hline Average Chlorine Dosage & $10 \mathrm{mg} / \mathrm{L}$ \\
\hline Maximum Chlorine Dosage & $20 \mathrm{mg} / \mathrm{L}$ \\
\hline Chlorinator Capacity & $1478 \mathrm{~kg} /$ day \\
\hline Chemical & Bleaching (NaClO) \\
\hline
\end{tabular}




\subsection{Gravity Thickener}

From concentration profile of sludge stream based on simulation results,

Total primary sludge, $Q_{P S}=1600 \mathrm{~m}^{3} / \mathrm{d}$ and total waste activated sludge, $Q_{\text {WAS }}=6896 \mathrm{~m}^{3} / \mathrm{d}$

Total mixed sludge influent flow, $Q_{M S}=Q_{P S}+Q_{W A S}=1600+6896=8496 \mathrm{~m}^{3} / d$

Solids load in the influent sludge, $S_{\text {TSS }}=73570 \mathrm{~kg} / \mathrm{d}$. [From simulation]

Solids loading rate for mixed sludge $=25-80 \mathrm{~kg} T S / \mathrm{m}^{2}$.day (Qasim, 2017)

So, assuming solids loading rate, $\mathrm{SLR}=60 \mathrm{~kg} \mathrm{TS} / \mathrm{m}^{2}$.day and removal efficiency $=90 \%$

Area, $\mathrm{A}=\frac{S_{T S S}}{S L R}=\frac{73570}{57}=1226.2 \mathrm{~m}^{2}$

Hydraulic loading rate, $\mathrm{HLR}=\frac{8496}{1226.2}=6.93 \mathrm{~m}^{3} / \mathrm{m}^{2}$.day

20-30 $\mathrm{m}^{3} / \mathrm{m}^{2}$. $\mathrm{d}$ HLR is recommended for gravity thickener. (Andreoli et al., 2007)

Assuming, $\mathrm{HLR}=20 \mathrm{~m}^{3} / \mathrm{m}^{2}$.day

So, dilution water needed, $\mathrm{Q}_{\mathrm{W}}=(H L R \times A)-Q_{M S}=(20 \times 1226.2)-8496=16028 \mathrm{~m}^{3}$

Total flow $=8496+16028=24524 \mathrm{~m}^{3}$

This flow increment does not affect the thickener performance. (Jordão e Pessôa, 1995)

Diameter, $\mathrm{D}=\sqrt{\frac{\overline{4 A}}{\Pi}}=\sqrt{\frac{4 \times 1226.2}{\Pi}}=39.5 \sim 40 \mathrm{~m}$

New area for the diameter, $\mathrm{A}_{\mathrm{n}}=\frac{\Pi \times D^{2}}{4}=\frac{3.1416 \times 40^{2}}{4}=1256.64 \mathrm{~m}^{2}$

Minimum side wall depth $3 \mathrm{~m}$ and maximum hydraulic retention time 24 hours are needed to avoid septic condition. (ABNT, 1989)

Assuming side wall height, $\mathrm{H}=3 \mathrm{~m}$

Bottom slope factor, $S_{F}=0.083$ (Metcalf et al., 2003)

Bottom slope, $\mathrm{S}_{\mathrm{L}}=H \times S_{F}=3 \times 0.083=0.25 \mathrm{~m}$

Center height, $\mathrm{H}_{\mathrm{C}}=H+S_{L}=3+0.25=3.25 \mathrm{~m}$ 
Volume, $\mathrm{V}=A \times H=1256.64 \times 3=3769.92 \mathrm{~m}^{3}$

Hydraulic retention time (HRT) without dilution, $T=\frac{3769.92}{8496}=0.44$ day $=10.6$ hours

Hydraulic retention time (HRT) with dilution, $T_{D}=\frac{3769.92}{24524}=0.15$ day $=3.6$ hours

Specification of thickener is shown in Table 10.

Table 10

Specification of gravity thickener

\begin{tabular}{|ll|}
\hline Parameter & Value \\
\hline Quantity & 1 No. \\
\hline Flow Rate with Dilution & $24.52 \mathrm{MLD}$ \\
\hline Detention Time & 3.6 hours \\
\hline Surface Area & $1256.64 \mathrm{~m}^{2}$ \\
\hline Diameter & $40 \mathrm{~m}$ \\
\hline Side Wall Height & $3 \mathrm{~m}$ \\
\hline Center Height & $3.25 \mathrm{~m}$ \\
\hline Free Board & $0.5 \mathrm{~m}$ \\
\hline Removal efficiency & $90 \%$ \\
\hline
\end{tabular}

\subsection{Anaerobic Sludge Digester}

Detention time 18-25 days and volumetric organic load 0.8-1.6 kg VSS/m³.day. (CIWEM, 1996)

From simulation results,

Total influent sludge flow, $Q_{T}=1550 \mathrm{~m}^{3} /$ day, solid load, $S_{T S S}=66710 \mathrm{~kg} \mathrm{TSS} / \mathrm{m}^{3}$.day and TSS $/ \mathrm{VSS}$ ratio, $r=0.65$ [From simulation]

Volatile solids load, $\mathrm{S}_{\mathrm{VSS}}=S_{T S S} \times r=66710 \times 0.65=43361.5 \mathrm{~kg} \mathrm{VSS} /$ day

Assuming volatile solids loading rate, $S_{\text {LRS }}=1.4 \mathrm{~kg} \mathrm{VSS} / \mathrm{m}^{3}$. day.

Volume, $\mathrm{V}=\frac{S_{V S S}}{S L R_{V S S}}=\frac{43361.5}{1.4}=30972.5 \mathrm{~m}^{3}$ 
15\% extra volume should be reserved for bio-gas accumulation. (Andreoli et al., 2007)

Headspace volume for gas, $\mathrm{V}_{\mathrm{G}}=V \times 0.15=30792.5 \times 0.15=4618.9 \mathrm{~m}^{3}$

Total volume, $\mathrm{V}_{\mathrm{T}}=V+V_{G}=30972.5+4618.9=35618.4 \mathrm{~m}^{3}$

Hydraulic detention time, $\mathrm{T}=\frac{V}{Q_{T}}=\frac{30972.5}{1550}=20$ days.

Assuming number of single-stage high-rate digesters, $\mathrm{N}=2$ Nos.

Flow rate for each digester, $\mathrm{Q}=\frac{Q}{N}=\frac{1550}{2}=775 \mathrm{~m}^{3} / \mathrm{d}$

Assuming height, $\mathrm{H}=15 \mathrm{~m}$

Surface area, $\mathrm{A}=\frac{V / N}{H}=\frac{35618.4 / 2}{15}=1187.3 \mathrm{~m}^{2}$

Diameter, $\mathrm{D}=\sqrt{\frac{\overline{4 A}}{\Pi}}=\sqrt{\frac{4 \times 1187.3}{\Pi}}=38.88 \mathrm{~m} \sim 39 \mathrm{~m}$

Diameter to height ratio $=\frac{39}{15}=2.6$

Bottom slope factor, $S_{F}=0.083$ (Metcalf et al., 2003)

Bottom slope, $\mathrm{S}_{\mathrm{L}}=H \times S_{F}=15 \times 0.083=1.25 \mathrm{~m}$

Center height, $\mathrm{H}_{\mathrm{C}}=H+S_{L}=15+1.25=16.25 \mathrm{~m}$

Specification of anaerobic digesters are shown in Table 11. 
Table 11

Specification of anaerobic digester

\begin{tabular}{|ll|}
\hline Parameter & Value \\
\hline Quantity & 2 Nos. \\
\hline Flow Rate & $775 \mathrm{MLD}$ \\
\hline Detention Time & 20 days \\
\hline Volume & $30972.5 \mathrm{~m}^{3}$ \\
\hline Headspace Volume for Gas & $4618.9 \mathrm{~m}^{3}$ \\
\hline Diameter & $39 \mathrm{~m}$ \\
\hline Side Wall Height & $15 \mathrm{~m}$ \\
\hline Center Height & $16.25 \mathrm{~m}$ \\
\hline
\end{tabular}

\subsection{Belt Press Filter}

Total influent flow to filter, $Q_{T}=1550 \mathrm{~m}^{3} /$ day and solids content, $C_{S}=2.09 \%$

Assuming number of belt press filters, $\mathrm{N}=4$ Nos.

Flow rate for each filter, $\mathrm{Q}=\frac{Q}{N}=\frac{1550}{4}=387.5 \mathrm{~m}^{3} / \mathrm{d}$

Assuming,

Solid capture, $\eta=95 \%$, specific gravity of sludge, $S_{S}=1.03$, cake solids content, $S_{C}=22 \%$ and nominal belt capacity, $C_{B}=320 \mathrm{~kg}$ TSS $/ \mathrm{m}$.hour (Metcalf et al., 2003)

Assuming operation hour, $T_{H}=12$ hours/day and operation day, $T_{D}=7$ days/week.

Wet solids load, $\mathrm{S}_{\mathrm{W}}=Q \times T_{D} \times S G_{S} \times 1000=387.5 \times 7 \times 1030=2793.88$ ton $/$ week

Dry solids load, $\mathrm{S}_{\mathrm{D}}=S_{W} \times C_{S}=2793.88 \times 0.0209=58.39$ ton $/$ week

Hourly solids rate, $\mathrm{S}_{\mathrm{DH}}=\frac{S_{D}}{T_{D^{\times}} T_{H}}=\frac{58390}{7 \times 12}=695.12 \mathrm{~kg} / \mathrm{hour}$.

Belt width, $\mathrm{W}=\frac{S_{D H}}{C_{B}}=\frac{695.12}{320}=2.17 \mathrm{~m} \sim 2.2 \mathrm{~m}$

Specification of belt press filters are shown in Table 12 . 
Table 12

Specification of belt press filter

\begin{tabular}{|ll|}
\hline Parameter & Value \\
\hline Quantity & 4 Nos. \\
\hline Flow Rate & $387.5 \mathrm{MLD}$ \\
\hline Operation Hour & 12 hours in a day \\
\hline Operation Day & 7 days in a week \\
\hline Belt Capacity & $320 \mathrm{~kg}$ TSS/m.hour \\
\hline Belt Width & $2.2 \mathrm{~m}$ \\
\hline Removal Efficiency & $95 \%$ \\
\hline
\end{tabular}

\section{Modeling And Simulation}

Every design should be modeled and simulated to check the result whether it should be implemented or not. The process of the STP was modeled in the GPS-X software version 8.0.1. The software was developed by Hydromantis Environmental Software Solutions, Inc. which is used to simulate the Wastewater, Water, Petrochemical wastewater treatment processes. The process was modeled under the comprehensive library (Carbon, Nitrogen, Phosphorus, $\mathrm{pH}$ ). The average temperature of Dhaka is around $30^{\circ}$ Celsius. Therefore, the plant was modeled under the average temperature and the flow load was taken for the maximum capacity of the plant. The model was simulated for a day to check model. All kinetics parameters were used as default values. The parameters which were considered for the design calculation were only used as input values and the rest parameters were taken as default values. Fig. 3 shows the process flow diagram (PFD) of the sewage treatment plant developed in the GPS-X.

All the equipment was modeled according to their specification and the simulation was done under steady-state conditions. A Sankey diagram is shown in Fig. 4 to represent the flow rate of various streams. The flow rate is shown in $\mathrm{m}^{3} /$ day unit. The composition of different parameters was analyzed from the outflow of all unit operations. Table 13 represents the flow rate and the composition of the different parameters (TSS, VSS, BOD, TN) for different streams that are used to design the sludge handling unit operations. The outfall quality is better and it meets the requirement of the Department of Environment (DoE), Bangladesh to discharge through the river.

The amount of treated water is $271.1 \mathrm{MLD}$ with a concentration of $32.28 \mathrm{mg} / \mathrm{L} \mathrm{TSS}, 14.87 \mathrm{mg} / \mathrm{L} \mathrm{BOD}$ and $60.24 \mathrm{mg} / \mathrm{L} \mathrm{TN}$. No operation in the process will reduce the TDS of the water. Therefore, the TDS will be the same as influent. The influent of the model is bod-based. The influent directly depends on the BOD and TN. The bar screen chamber reduces a little TSS and the chlorination chamber does not reduce the concentration of the contaminants except pathogen concentration, so they are not shown in the table. 
Table 13

Flowrate and composition of TSS, VSS, BOD, TN for different streams

\begin{tabular}{|llllll|}
\hline Stream & Flowrate $\left(\mathrm{m}^{3} / \mathrm{d}\right)$ & $\begin{array}{l}\text { TSS } \\
(\mathrm{mg} / \mathrm{L})\end{array}$ & $\begin{array}{l}\text { VSS } \\
(\mathrm{mg} / \mathrm{L})\end{array}$ & $\begin{array}{l}\text { BOD } \\
(\mathrm{mg} / \mathrm{L})\end{array}$ & $\begin{array}{l}\text { TN } \\
(\mathrm{mg} / \mathrm{L})\end{array}$ \\
\hline Influent & 218700 & 361.50 & 271.10 & 350.00 & 70.00 \\
\hline Equalization Tank Outlet & 73900 & 358.10 & 264.10 & 333.10 & 78.90 \\
\hline Grit Chamber Outlet & 73900 & 348.10 & 264.10 & 333.10 & 78.90 \\
\hline Primary Clarifier Outlet & 73500 & 322.10 & 244.90 & 318.40 & 75.15 \\
\hline Recycled Activated Sludge & 3448 & 8457.00 & 5098.00 & 3473.00 & 570.40 \\
\hline Aeration Tank Outlet & 40200 & 935.60 & 564.00 & 385.70 & 114.90 \\
\hline Secondary Clarifier Outlet & 71780 & 32.28 & 19.46 & 14.87 & 60.24 \\
\hline Primary Sludge (PS) & 400 & 5183.00 & 3939.00 & 3342.00 & 642.90 \\
\hline Waste Activated Sludge (WAS) & 1724 & 8457.00 & 5098.00 & 3473.00 & 570.40 \\
\hline Total Sludge with Water & 24520 & 2737.00 & 1703.00 & 1204.00 & 241.70 \\
\hline Thickened Sludge & 1550 & 39250.00 & 24430.00 & 17150.00 & 2706.00 \\
\hline Thickener Supernatant & 22970 & 273.70 & 170.30 & 128.70 & 75.47 \\
\hline Digester Outlet & 775 & 20200.00 & 6065.00 & 1718.00 & 2649.00 \\
\hline Total Filtrate & 1414 & 1010.00 & 303.30 & 96.01 & 2009.00 \\
\hline Outfall & 271100 & 32.28 & 19.46 & 14.87 & 60.24 \\
\hline
\end{tabular}

From Table 14, the removal efficiency of primary clarifiers for TSS, BOD and TN are $7.5 \%, 4.4 \%$ and $4.8 \%$ respectively. The removal efficiency of the primary clarifier depends on the underflow rate of the clarifier. Therefore, it varies with the sludge production rate. Aeration tank removal efficiency for TSS, BOD and TN depends on the airflow rate and the flow of activated sludge. The effluent quality will not be better without supplying an adequate air flow rate. A secondary clarifier has higher removal efficiency than any other treatment operation. Primary sludge has a solid content of $0.52 \%$ and waste activated sludge has a solids content of $0.85 \%$. The thickened sludge solid content is $3.9 \%$ and the digested sludge solids content is $2 \%$. The TSS, VSS and BOD reduction efficiencies of the anaerobic digester are $48.5 \%, 75.2 \%$ and $90 \%$ respectively. The maximum specific growth rate of heterotrophic bacteria, methylotrophs, ammonia oxidizer, nitrate oxidizer, anammox bacteria, poly-phosphate accumulating oxidizer (PAO) and acetogenic bacteria are $3.21 / \mathrm{d}, 1.31 / \mathrm{d}, 0.91 / \mathrm{d}, 1.01 / \mathrm{d}, 0.021 / \mathrm{d}, 1.01 / \mathrm{d}$ and $0.351 / \mathrm{d}$ respectively. The fermentation and the hydrolysis rate are $3.01 / \mathrm{d}$. In the calculation, the underflow rate and the BOD 
removal efficiency of the primary clarifier are neglected to keep the process performance steady and better.

Table 14

Removal efficiency of different parameters for different unit operations

\begin{tabular}{|lllll|}
\hline Equipment & TSS (\%) & VSS (\%) & BOD (\%) & TN (\%) \\
\hline Grit Chamber & 2.8 & 0.0 & 0.0 & 0.0 \\
\hline Primary Clarifier & 7.5 & 7.3 & 4.4 & 4.8 \\
\hline Aeration Tank & 15.3 & 21.3 & 39.6 & 9.9 \\
\hline Secondary Clarifier & 96.5 & 96.5 & 96.1 & 47.6 \\
\hline Thickener & 90.0 & 90.0 & 89.3 & 68.8 \\
\hline Anaerobic Digester & 48.5 & 75.2 & 90.0 & 2.1 \\
\hline Belt Press Filter & 95.0 & 95.0 & 94.4 & 24.2 \\
\hline
\end{tabular}

\section{Conclusion}

A sewage treatment plant is necessary to keep our environment clean and safe. The process selection and unit operations depend on the influent characteristics of the sewage. This plant process was designed for the discharge guideline of the ECR 97, Bangladesh. Different country has different guideline for the discharge to the environment. Therefore, the process and unit operations may vary. The climate of Bangladesh is warm and humid. Therefore, the diurnal flow factor was considered in the design phase of the plant. The population load and the peak factor should be calculated precisely to design the equipment. Inaccurate information of the capacity will lead to plant failure severely.

The simulation result depends on the library and the input parameters. Therefore, the data should be input into the model carefully. Some input was set as default in this simulation. The assumptions of some parameters in the design calculation were done from the typical data range of existing plants. The removal efficiency of the equipment depends on the detention time and the reaction kinetics. Students, academics and young engineers will be able to design and model a new STP from the paper. The government should come forward to implement some sewage treatment plants to keep the environment safe.

\section{Declarations}

\section{DECLARATION OF COMPETING INTEREST}


I do declare that I have no known competing financial interests or personal relationships that could have appeared to influence the work reported in this paper. This paper is not in any other publication process. This research did not receive any specific grant from funding agencies in the public, commercial, or notfor-profit sectors.

\section{ACKNOWLEDGEMENT}

The author is grateful to Antora Yasmin, who assisted me in writing. He is also thankful to his colleagues Engr. Bipul Kumar, Engr. Abdur Razzaq and Engr. Sajib Ali for proofreading the paper.

\section{References}

Abdel-Raouf, N., Al-Homaidan, A. A., \& Ibraheem, I. (2012). Microalgae and wastewater treatment. Saudi journal of biological sciences, 19(3), 257-275.

ABNT (1989). "Projeto de esta, $c^{\sim}$ oes de tratamento de esgotos" [Design of Sewage Treatment Plants] [In Portuguese], NB 570, Associa, $c^{\sim}$ ao Brasileira de Normas T'ecnicas.

Andreoli, C. V., Von Sperling, M., \& Fernandes, F. (2007). Sludge treatment and disposal. IWA publishing. BBS (Bangladesh Bureau of Statistics) 2003. Population Census 2001.

BNBC (Bangladesh National Building Code) 2020. Government of The People's Republic of Bangladesh. Cheremisinoff, N. P. (2001). Handbook of water and wastewater treatment technologies. ButterworthHeinemann.

CIWEM (The Chartered Institution of Water and Environmental Management) (1996) Sewage sludge Utilization and disposal. In Handbook of UK Wastewater Practice, 110 pp.

Crini, G., \& Lichtfouse, E. (2019). Advantages and disadvantages of techniques used for wastewater treatment. Environmental Chemistry Letters, 17(1), 145-155.

DoE, Bangladesh Standard (Department of Environment) 1997. The environment conservation rules 1997.

Droste, R. L., \& Gehr, R. L. (2018). Theory and practice of water and wastewater treatment. John Wiley \& Sons.

Eckenfelder Jr, W. W., \& Updated by Staff. (2000). Wastewater treatment. Kirk-Othmer Encyclopedia of Chemical Technology.

Gebara, F. (1999). Activated sludge biofilm wastewater treatment system. Water Research, 33(1), 230-238. 
Jord a ao, E.P., Pessoa, C.A. (1995) Tratamento de esgotos dom'esticos [Domestic Sewage Treatment] [In Portugese]. ABES, 3rd edn, p. 683.

Kodavasal, A. S. (2011). The STP Guide. Design, operation, maintenance. Karnataka Pollution Control Board. Available on http://kspcb. gov. in/STP-Guide-web\% 28Med, 29.

Kumar, K., Kumar, P., \& Babu, M. (2010). Performance evaluation of waste water treatment plant. International Journal of Engineering Science and Technology, 2(12), 7785-7796.

Kumar, P. R., Pinto, L. B., \& Somashekar, R. K. (2010). Assessment of the efficiency of sewage treatment plants: a comparative study between nagasandra and mailasandra sewage treatment plants. Kathmandu university journal of science, engineering and technology, 6(2), 115-125.

LaGrega, M. D., \& Keenan, J. D. (1974). Effects of equalizing wastewater flows. Journal (Water Pollution Control Federation), 123-132.

Lazarova, V., Savoye, P., Janex, M. L., Blatchley lii, E. R., \& Pommepuy, M. (1999). Advanced wastewater disinfection technologies: state of the art and perspectives. Water Science and Technology, 40(4-5), 203213.

Libralato, G., Ghirardini, A. V., \& Avezzù, F. (2012). To centralise or to decentralise: An overview of the most recent trends in wastewater treatment management. Journal of environmental management, 94(1), 6168.

Mahmoud, A., Olivier, J., Vaxelaire, J., \& Hoadley, A. F. (2013). Advances in mechanical dewatering of wastewater sludge treatment. In Wastewater Reuse and Management (pp. 253-303). Springer, Dordrecht.

Mara, D. (2013). Domestic wastewater treatment in developing countries. Routledge.

Marco, A., Esplugas, S., \& Saum, G. (1997). How and why combine chemical and biological processes for wastewater treatment. Water Science and Technology, 35(4), 321-327.

Massoud, M. A., Tarhini, A., \& Nasr, J. A. (2009). Decentralized approaches to wastewater treatment and management: applicability in developing countries. Journal of environmental management, 90(1), 652659.

Metcalf, L., Eddy, H. P., \& Tchobanoglous, G. (1991). Wastewater engineering: treatment, disposal, and reuse (Vol. 4). New York: McGraw-Hill.

Mittal, A. (2011). Biological wastewater treatment. Water Today, 1, 32-44.

Patterson, J. W. (1985). Industrial wastewater treatment technology.

Pescod, M. B. (1992). Wastewater treatment and use in agriculture-FAO irrigation and drainage paper 47. Food and Agriculture Organization of the United Nations, Rome. 
Qasim, S. R. (2017). Wastewater treatment plants: planning, design, and operation. Routledge.

Russell, D. L. (2019). Practical wastewater treatment. John Wiley \& Sons.

Samal, S. S. (2016). Design of Sewage Treatment Plant.

Sawicki, J. M. (2004). Aerated grit chambers hydraulic design equation. Journal of Environmental Engineering, 130(9), 1050-1058.

Schroeder, E. D. (1977). Water and wastewater treatment. McGraw-Hill, New York N. Y. 1977. $370,3$.

Sonune, A., \& Ghate, R. (2004). Developments in wastewater treatment methods. Desalination, 167, 55-63.

Speece, R. E. (1983). Anaerobic biotechnology for industrial wastewater treatment. Environmental science \& technology, 17(9), 416A-427A.

Varadi, L. (1984). Inland Aquaculture Engineering. Food and Agriculture Organization.

Vesilind, P. (Ed.). (2003). Wastewater treatment plant design (Vol. 2). IWA publishing.

\section{Figures}

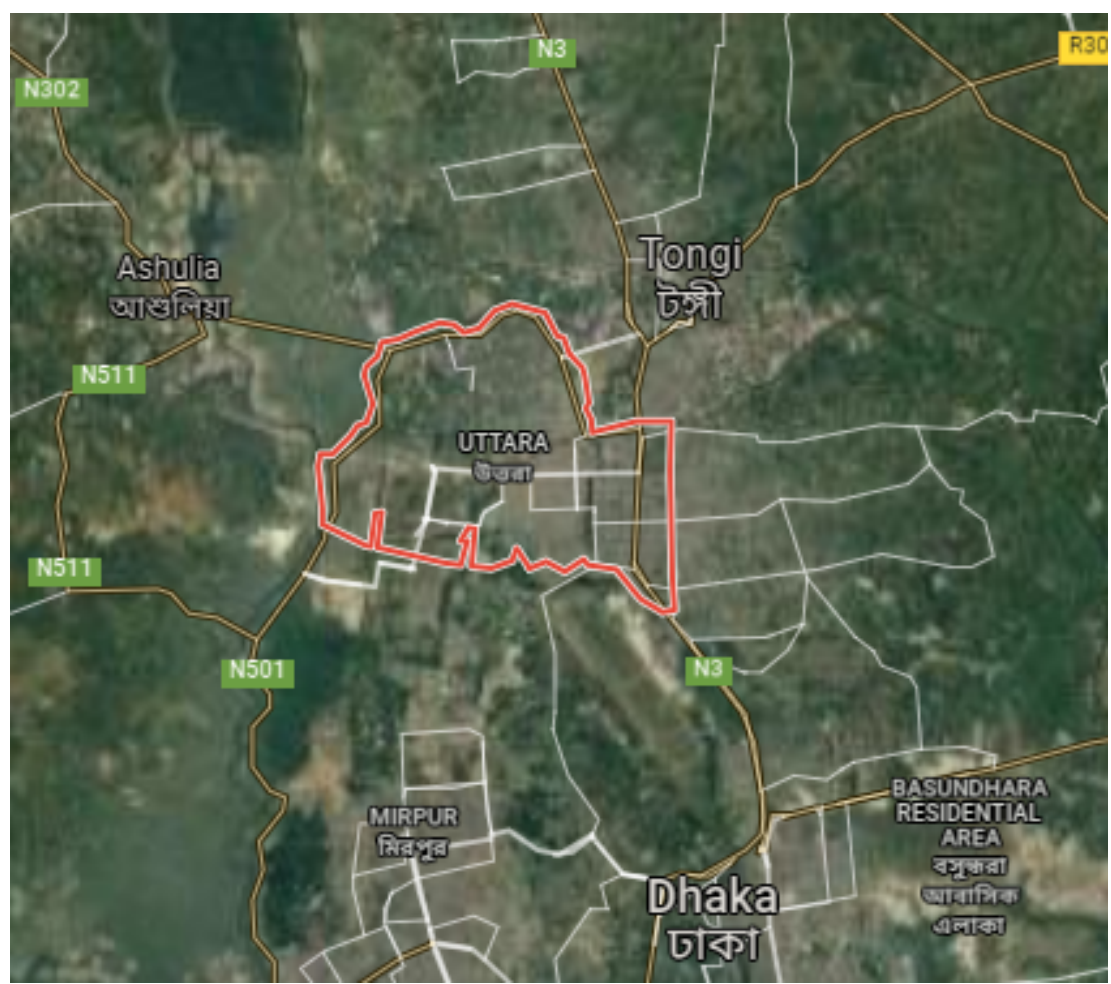

\section{Figure 1}

Satellite map of Uttara City, Dhaka, Bangladesh 


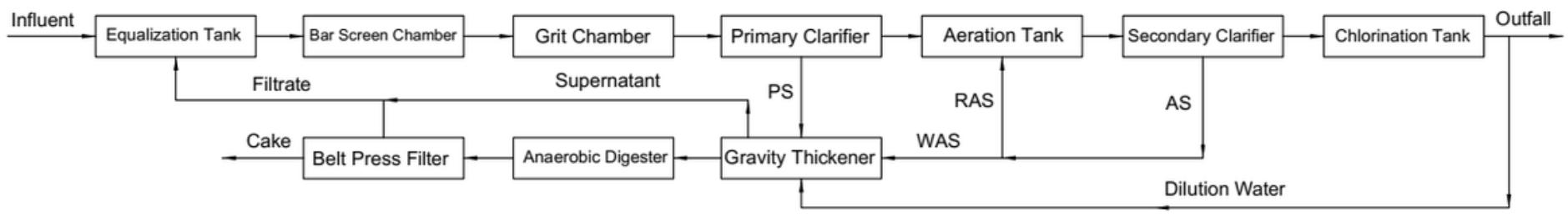

Figure 2

Process Block Diagram (PBD) of the STP

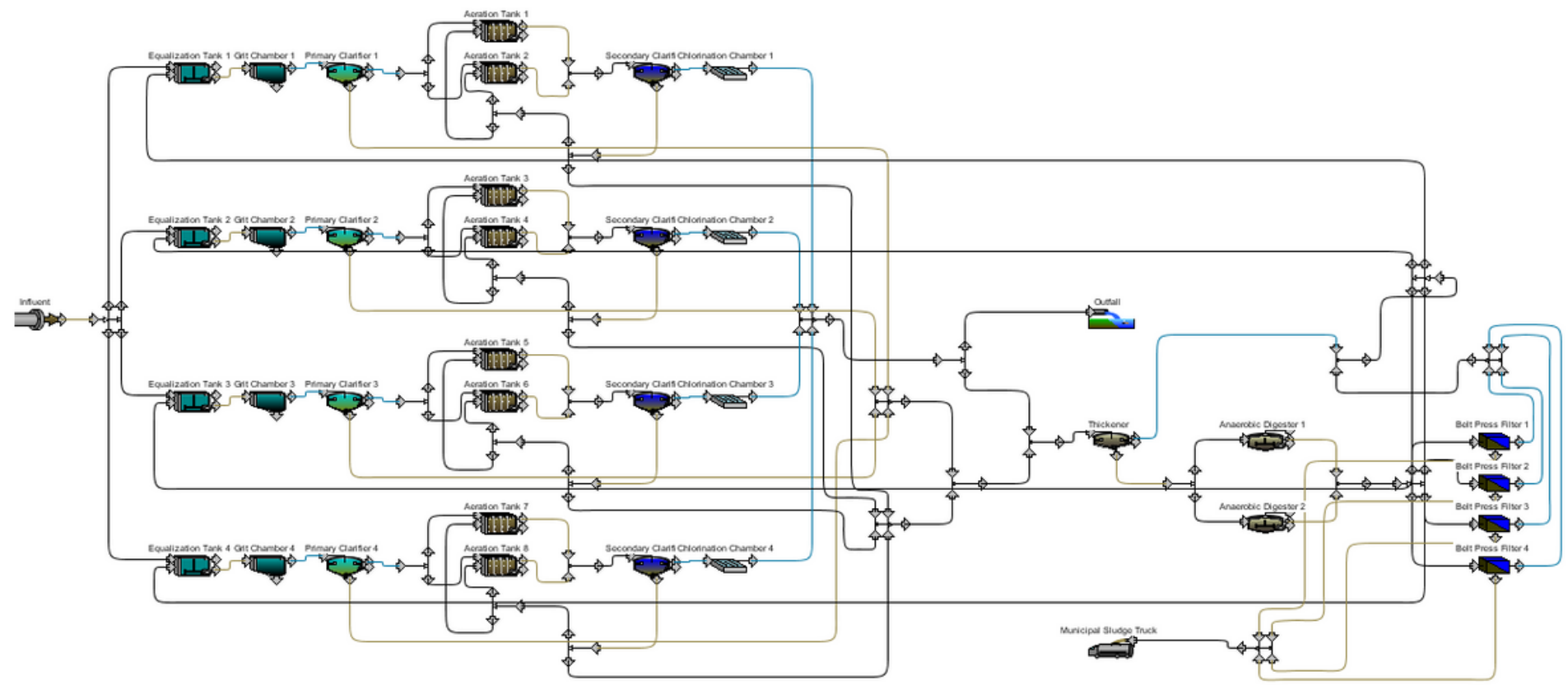

Page 27/28 
Figure 3

Process flow diagram (PFD) of the STP

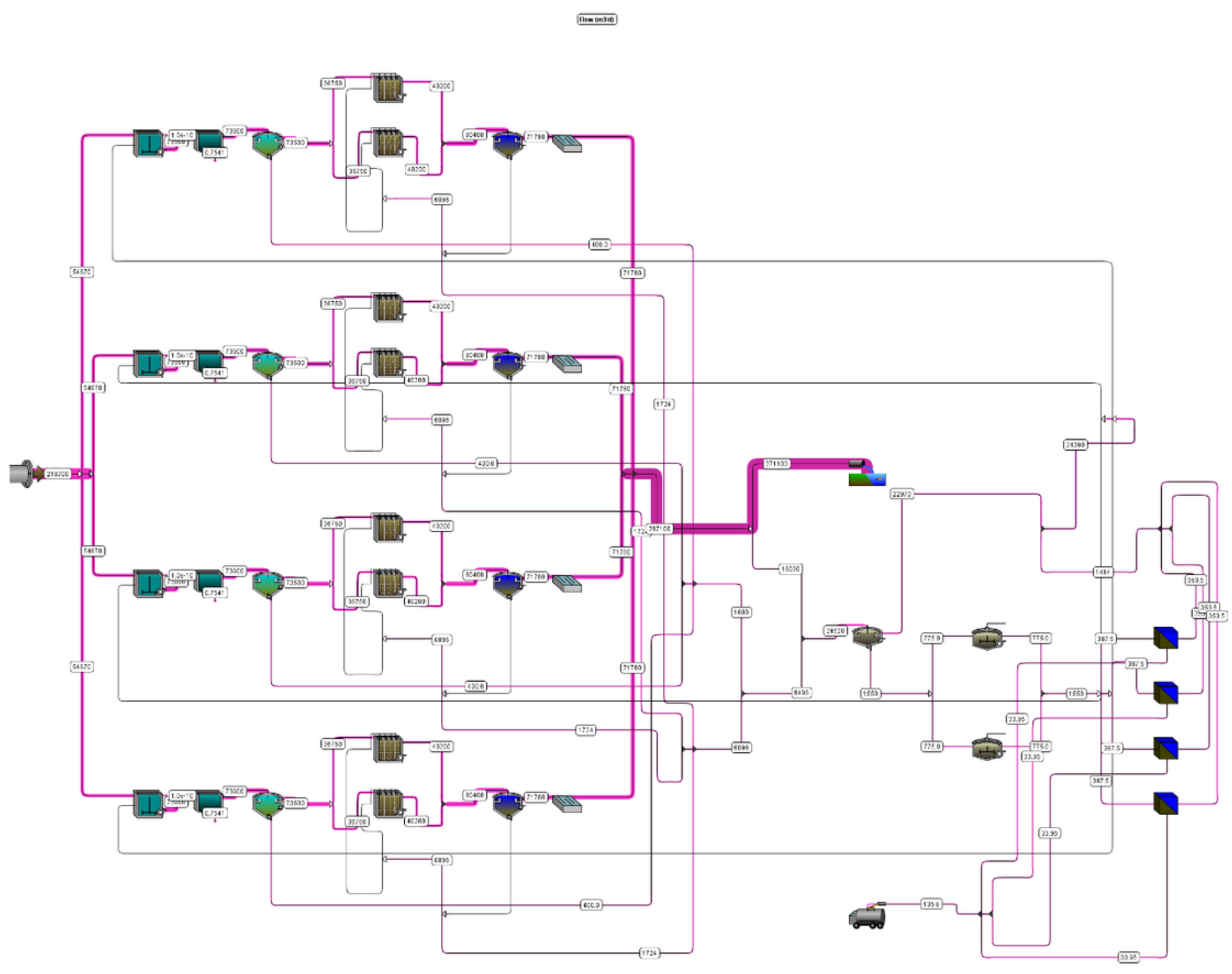

Figure 4

Sankey diagram of the STP with flowrate $\left(\mathrm{m}^{3} / \mathrm{d}\right)$ 